\title{
Subsea Cable Condition Monitoring with Distributed Optical Fibre Vibration Sensor
}

\author{
Ali Masoudi, James A. Pilgrim, Trevor P. Newson, and Gilberto Brambilla
}

\begin{abstract}
A novel subsea cable condition monitoring technique based on embedded optical fibre inside the cable is demonstrated. It is shown that a distributed optical fibre vibration sensor can be used to map dynamic strains all along the cable simultaneously. It is experimentally shown that such system can fully quantify the location and strain level at each point on the cable as a function of time for both abrupt impact and cyclical loading. The sensing system demonstrated a spatial and strain resolution of $1 \mathrm{~m}$ and $4 \mu \varepsilon$, respectively, over a $10 \mathrm{~km}$ sensing range.
\end{abstract}

If All the data supporting this study are openly available from the University of Southampton repository at http://doi.org/10.5258/SOTON/????. Index Terms-Distributed Vibration Sensor (DVS), Subsea cable monitoring, Offshore wind farm, Submarine cable installation, Cable fault monitoring, Distributed Acoustic Sensor (DAS).

\section{INTRODUCTION}

$\mathbf{O}$ FFSHORE wind energy plays an important role in the expansion and diversification of the renewable energy generation worldwide [1], [2]. The cost of lost wind farm production due to subsea cable failure, however, is a major issue in this sector [3]. Between 2002 and 2015, the insurance losses due to cable failure were more than $£ 140$ million in the UK alone [4]. Some failures are mechanical and occur due to damage sustained during the installation phase when the cable is more likely to experience over-bending or excessive tension. Such problems can occur due to insufficient coordination of the cable laying vessel's forward motion and cable payout mechanism, loss of dynamic positioning system of the vessel due to waves or currents preventing the vessel from keeping its position, and the cable hitting submarine debris on the seabed [5]. The main cause of cable failure after the installation is mechanical damages caused by human activities such as fishing and ship anchoring [6].

A condition monitoring system capable of mapping the curvature and tension of the cable in real time can reduce the instances of these faults, thus substantially increasing the reliability of offshore subsea cable systems [4]. Recent progress in distributed optical fibre sensing (DOFS) systems make them an ideal tool to monitor the condition of subsea

Manuscript received xxx xxx, 2018; revised xxx xxx, 2018; accepted xxx $\mathrm{xxx}, 2018$. Date of publication $\mathrm{xxx} \mathrm{xxx}, 2018$. This work is funded by Carbon Trust and EPSRC UK grant EP/N00437X/1. A. Masoudi and Gilberto Brambilla are with the Optoelectronics Research Centre (ORC), University of Southampton, Southampton SO17 1BJ, UK. (e-mail: a.masoudi@soton.ac.uk; tpn@soton.ac.uk; gb2@orc.soton.ac.uk). J. A. Pilgrim is with the School of Electronics and Computer Science, University of Southampton, Southampton SO17 1BJ, U.K. (e-mail: jp2@ecs. soton.ac.uk). Color versions of one or more of the figures in this paper are available online at http://ieeexplore.ieee.org. cables. A subcategory of this sensing technology known as distributed vibration sensors (DVSs) can be used for quantitative analysis of dynamic strains along subsea cables during both the installation process and operation in order to detect and quantify mechanical damages to the cable.

Distributed vibration sensing systems are based either on Brillouin or Rayleigh scattering [7], [8]. The application of Brillouin-based DVS is limited to measurement of low frequency perturbations over a relatively short sensing range [9][13]. The limited sensing and frequency range of Brillouinbased DVSs are due to the time-consuming data acquisition process in Brillouin-based sensors as well as a need for an extensive time-averaging at each frequency step to achieve a desirable signal to noise ratio (SNR). Furthermore, the strain resolution of Brillouin based DVS systems is limited to few micro-strains.

Rayleigh-based DVSs, on the other hand, are capable of mapping high frequency perturbations over a much longer sensing range and with a higher strain sensitivity [14] $-[19]$. In this study, a Rayleigh-based DVS based on optical fibre interferometry is used to assess the feasibility of monitoring mechanical stresses in subsea power cable using an optical fibre in the optical fibre bundle which can be found inside any three-core $\mathrm{AC}$ cables used by offshore wind farms. The main advantage of the DVS system employed in this study is its simplicity and cost-effectiveness.

\section{PRINCIPLES}

Mechanical stresses along a power cable can be measured by mapping the strain distribution along the optical fibre embedded inside the cable. To evaluate the strain distribution along the fibre, Phase-based Optical Time Domain Reflectometry $(\varphi$-OTDR) interrogation technique can be used. In this technique, the strain level at any given section of the fibre is measured by analysing the phase of the backscattered light from that section. To evaluate the strain level over a given section of the fibre with length $L$, as shown in figure 11(a), an optical pulse is launched into the fibre and the phase difference between the backscattered Rayleigh light from the two ends of that section is measured. For a cable under no mechanical stresses, the phase difference is given by [20]:

$$
\Delta \varphi=\varphi_{A}-\varphi_{B}=\frac{2 \pi n}{\lambda} 2 L+\phi
$$

where $\varphi_{A}$ and $\varphi_{B}$ are the phases of the backscattered light from the two ends, $n$ is the effective refractive index of the 
fibre, $\lambda$ is the wavelength of the light, and $\phi$ is a random phase. When the cable is bent, the distance between the two ends of that section of fibre increases or decreases depending on the bending moment. The new phase difference over the same section of the fibre is given by

$$
\Delta \varphi^{\prime}=\varphi_{A}^{\prime}-\varphi_{B}^{\prime}=\frac{2 \pi n}{\lambda} 2(L \pm \Delta L)+\phi^{\prime}
$$

where $\Delta L$ is the change in length and the plus and minus signs are used for the fibre under tension and compression, respectively. By comparing the phase difference before and after subjecting the cable to different deflections, the changes in the length of the fibre and, consequently, the strain of the cable can be determined. Dynamic strain variation along the cable can be mapped by continuously monitoring the changes in the phase of the backscattered light for each individual sections of the optical fibre.

A typical subsea HVAC cable consist of three conductors twisted together along with an optical fibre cable and protected by a metallic armour and an outer serving (figure 1(b)). In the strain analysis of such cable, the effect of the strain-free window of operation of the optical fibre bundle within the cable as well as the helical arrangement of the elements inside the cable serving need to be considered.

The optical fibre bundle inside a subsea cable has a strainfree window of operation in which cable elongation or contraction has little effect on the fibres inside the cable. The strainfree window is achieved by wounding the loose tube fibre around the central elements of the cable [21]. The curvature of the tube makes it possible for the fibres to find a shorter

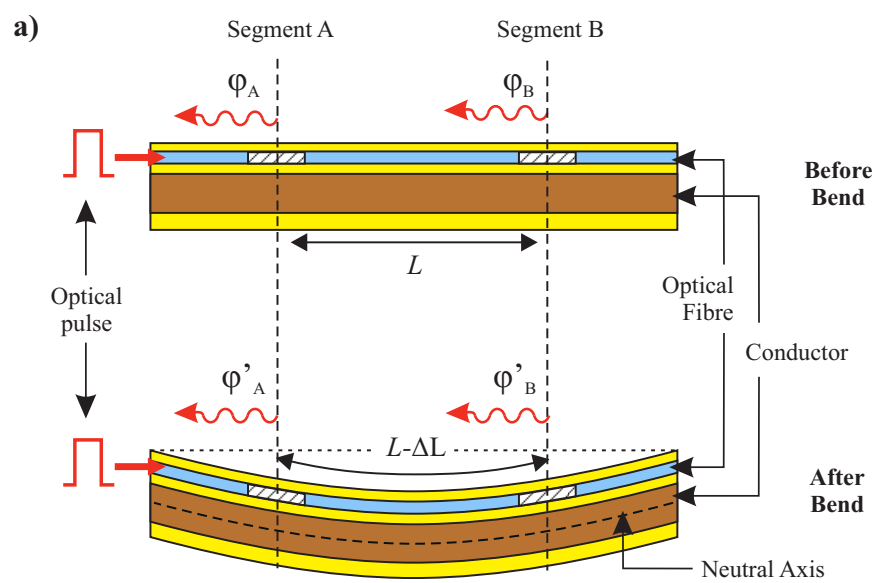

b)

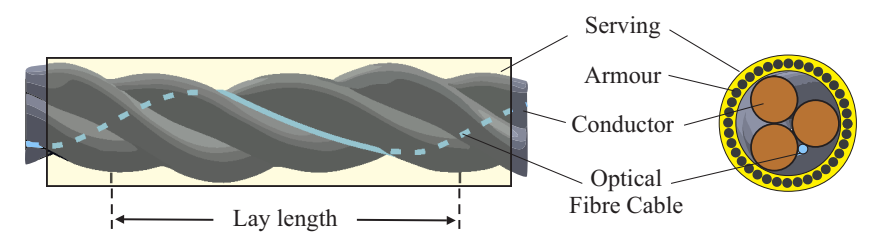

Figure 1. Principle of subsea cable dynamic strain measurement based on $\varphi$-OTDR. a) A simplified axial section area of a cable with embedded optical fibre before and after deflection. b) Cross section and axial section area of a three-phase submarine cable with embedded optical fibre demonstrating the helical cable elements and their lay length. path than the centre-line of the loose tube thereby avoiding elongation due to small deflection of cable. These provisions to mechanically decouple the optical fibres from other cable elements mean that, once the strain-free window is exhausted due to significant cable deformation, the optical fibres inside the cable experience a fraction of the total strain on the cable. The difference between the actual strain on the cable and the strain level experienced by the fibres needs to be determined experimentally.

The helical geometry of the fiber optic bundle inside the cable also affects the strain measurement. The level of strain imposed on an optical fibre depends on both the cable deflection and the position of the fibre bundle relative to the neutral axis of the cable (figure 2). Due to this geometry, the fibre experiences strains from both tensile force and bending moment. The strain level over an infinitesimal length $d x$ of the fibre is:

$$
\varepsilon_{\text {fibre }}=\varepsilon_{T}+\varepsilon_{b}
$$

where $\varepsilon_{T}$ and $\varepsilon_{b}$ are the tensile and bending strains, respectively. Since DVS systems measure the net fibre elongation $\Delta L$ over a predetermine gauge length $L$, fibre elongation can be calculated by integrating the strain over the gauge length:

$\Delta L\left(x_{0}\right)=\int_{x_{0}}^{x_{0}+L} \varepsilon_{f i b r e} d x=\int_{x_{0}}^{x_{0}+L} \varepsilon_{T} d x+\int_{x_{0}}^{x_{0}+L} \varepsilon_{b} d x$

where $x_{0}$ can be any point on the sensing fibre.

A cable with a helically wrapped subcomponents has a high axial strength at its principal loading direction combined with a substantial flexibility at all other loading directions. When such cable is deflected, the subcomponents within the cable experience a high bending strain because of the cable deformation due to normal loads while sustaining little tensile strain as no longitudinal force is applied. Therefore, in order to study the strains and stresses on the optical fibre due to cable deflection, tensile strain on the fibre can be neglected.

In order to measure the bending strain $\varepsilon_{b}$ on the optical fibre inside a deflected cable, the curvature of the cable needs to be calculated first. The curvature can be calculated by fitting a second order polynomial to the measured cable deflection, $\delta$, with the end points held at zero deflection. The fitted polynomial equation $\delta(x)$ is deemed accurate only if $R^{2}$ mean value of the fitted curve is greater than 0.9. The curvature can be calculated from the deflection as [22]:

$$
\kappa=\frac{\delta^{\prime \prime}}{\left(1+\delta^{\prime 2}\right)^{3 / 2}}
$$

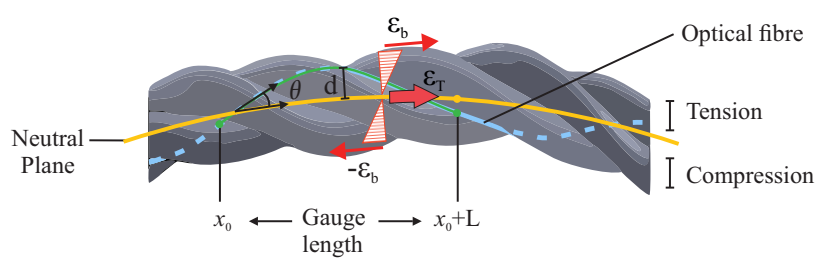

Figure 2. Strain distribution along an optical fibre cable inside a deflected subsea HVAC cable in which the cable elements are helically arranged. 
where $\delta^{\prime}$ and $\delta^{\prime \prime}$ are the first and second derivatives of $\delta$. The curvature of the cable can be used to calculate the maximum bending strain on the optical fibre that sits $d$ meter away from the principal axis of the cable using the following equation [23] :

$$
\varepsilon_{b, \max }=\kappa d \cos (\theta)
$$

where $\theta$ is the helix angle of the subcomponents within the cable. The net strain level on the optical fibre depends on the position of the fibre relative to the neutral plane of the cable as shown in figure 2. The strain level varies from $\varepsilon_{b, \max }$ for fibres experiencing elongation (top) to $-\varepsilon_{b, \max }$ for fibres experiencing compression (bottom). Therefore, the total elongation due to bending strain over the gauge length $L$ is given by:

$$
\begin{aligned}
\Delta L\left(x_{0}\right) & =\int_{x_{0}}^{x_{0}+L} \varepsilon_{b, \max } \sin \left(\frac{2 \pi}{L_{\text {lay }}} x\right) d x \\
& =\int_{x_{0}}^{x_{0}+L} \kappa d \cos (\theta) \sin \left(\frac{2 \pi}{L_{\text {lay }}} x\right) d x \\
& =d \cos (\theta) \int_{x_{0}}^{x_{0}+L} \kappa(x) \sin \left(\frac{2 \pi}{L_{\text {lay }}} x\right) d x
\end{aligned}
$$

where $L_{l a y}$ is the lay-length of the cable and $L$ is the gauge length of the DVS system (figure 1 (b)). This equation shows that fibre elongation depends on both the gauge length of the sensing system and the lay-length of the cable and it varies periodically along the sensing fibre. For instance, according to equation (7), if the gauge length of the DVS system is equal to the lay-length of the cable $\left(L=L_{l a y}\right)$, the elongation due to bending strain will be zero since the positive strain from the section of the fibre under tension will be canceled out by the negative strain from the next section of the fibre under compression. For a DVS system with a gauge length shorter than the cable lay-length, the elongation is the sum of the strains over the gauge length of the sensing system.

\section{EXPERIMENTAL ARRANGEMENT AND Procedure}

Figure 3 shows the experimental arrangement used to assess the capability of the DVS developed by our research team to monitor dynamic strains and impacts along subsea power cable. The arrangement had two main sections: an optical fibre interrogator and a hydraulic test rig.

\section{A. Optical Fibre Interrogator}

To interrogate the optical fibre inside the HVAC cable, a DVS sensing unit based on $\varphi$-OTDR was used [24]. The schematic diagram of the interrogation unit is shown in figure 3 a). A $1550 \mathrm{~nm}$ distributed feedback (DFB) laser was modulated to generate $8 n s$ optical pulse with a peak power of $20 \mathrm{~mW}$. The optical pulse was amplified by an Erbium-doped fibre amplifier (EDFA1) to enhance the peak power to $1 W$. The amplified pulse was passed through a dense wavelength division multiplexing (DWDM) filter $(100 \mathrm{GHz}$ bandwidth, a)
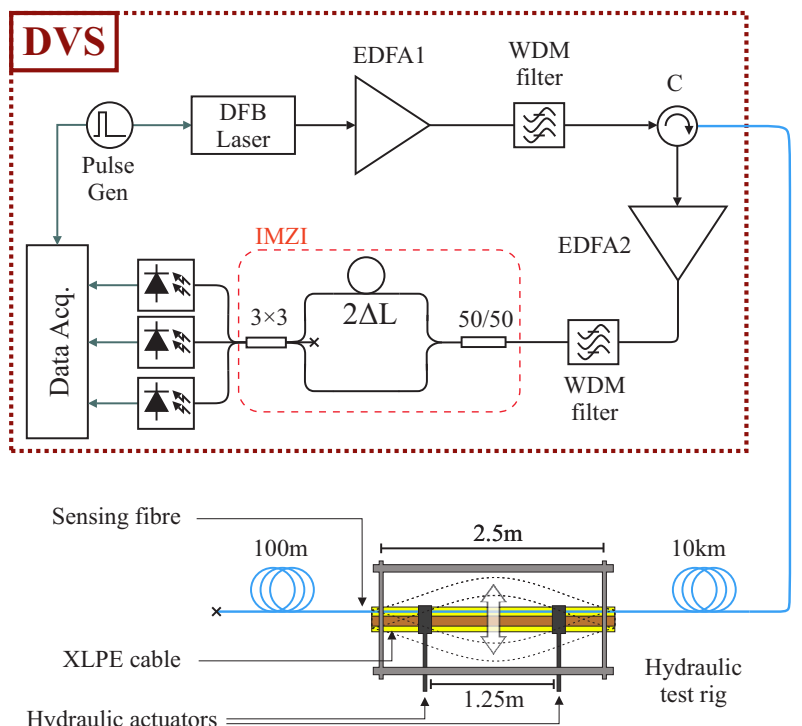

b)

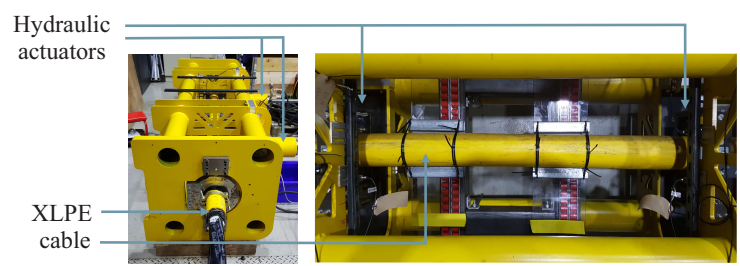

Figure 3. a) Experimental arrangement. DVS: Distributed Vibration Sensor; DFB Laser: Distributed feedback laser; EDFA: Erbium-doped fibre amplifier; WDM filter: Wavelength division multiplexing filter; C: Circulator; IMZI: Imbalanced Mach-Zehnder interferometer; Data Acq: Data acquisition system. b) Photos of the hydraulic test rig used to cyclically load the subsea cable.

ITU channel C34) to filter the amplified spontaneous emission (ASE) from the EDFA1. The amplified pulse was launched into the sensing fibre via a circulator $\mathrm{C}$.

The backscattered light from the sensing fibre was collection by the same circulator and amplified by a second optical amplifier (EDFA2) with $25 d B$ gain. The amplified backscattered light was passed through another DWDM filter to remove the ASE noise and launched into an imbalanced Mach-Zehnder interferometer (IMZI). The role of the IMZI was to extract the phase information from the backscattered light [24]. The path imbalance of the IMZI was set to $2 m$ to achieve $1 m$ spatial resolution. A symmetrical $3 \times 3$ coupler was used at the output of the IMZI to avoid phase signal fading. The IMZI was thermally and acoustically isolated to prevent any undesirable changes in the path difference.

The three output arms of the IMZI were connected to three photoreceivers $(125 \mathrm{MHz}$ bandwidth, $40 \mathrm{~V} / \mathrm{mA}$ transimpedance amplification, $22.5 \mathrm{pW} / \sqrt{\mathrm{Hz}}$ Noise equivalent power) and sampled at $500 \mathrm{MSa} / \mathrm{s}$ by a high speed data acquisition (DAQ) card. The acquired data was then processed to extract the phase information along the sensing fibre. In order to eliminate $1 / f$ noise in the system, the phase information at each sampling point was filter digitally by a band-pass filter (BPF) with a passband from $0.1 \mathrm{~Hz}$ to $500 \mathrm{~Hz}$. 


\section{B. Hydraulic Test Rig}

The hydraulic test rig consisted of a $2.5 \mathrm{~m}$ steel frame and two hydraulic actuators to exert load on the subsea cable (figure 3(b)) as per a typical four point bending test. The test rig was designed to support the cable at two points $2.5 \mathrm{~m}$ apart, where it was allowed to freely pivot and slide in translation. The two hydraulic actuators were installed $625 \mathrm{~mm}$ away from each end of the test rig. In this study, a $3.5 \mathrm{~m}$ long three-phase $33 \mathrm{kV}$ armored cable which is typically used in wind farm arrays was used as a test bench. The lay-length of the cable cores was $\sim 1.6 \mathrm{~m}$ and the helix angle of its subcomponents was $16^{\circ}$. Two hydraulic actuators were used to cyclically deflect the cable between $\pm 50 \mathrm{~mm}$ and $\pm 150 \mathrm{~mm}$. To evaluate the sensing range of the system a $\sim 10 \mathrm{~km}$ dummy optical fibre was added between the sensing unit and the test rig. Another $100 \mathrm{~m}$ of dummy fibre was added to the end of the fibre within the test cable to separate the end of the sensing fibre from the cable under test. The dummy fibres were isolated from external temperature and strain fluctuations.

\section{Experimental Procedure}

The aim of this experiment was to determine the capability of the DVS system to measure cyclical loading and abrupt impact on subsea cables. For the cyclical loading test, the cable was deflected periodically by the hydraulic actuators with three traveling distances of $\pm 50 \mathrm{~mm}, \pm 100 \mathrm{~mm}$, and $\pm 150 \mathrm{~mm}$ about the mid point. Before the test, the cable deflection was evaluated by measuring the cable displacement at five points as shown figure 4 The cable deflection was used to calculate the curvature of the cable.

For the impact test, a $4 \mathrm{~kg}$ wedge shaped steel block with a $90^{\circ}$ angle was dropped on the middle of the test cable from a height of $114 \mathrm{~cm}$. This test was a standard impact test established by European Committee for Electrotechnical Standardization (test standard EN61442). For this assessment, the same test rig and test cable were used but the hydraulic actuators were removed in order not to restrict the cable.

The pulse repetition period for both cyclical loading and impact tests was set to $900 \mu \mathrm{s}$ with a data acquisition time of $60 s$ for cyclical loading and $20 s$ for impact test.

\section{EXPERIMENTAL RESUlts}

Figure 5 shows the experimental results obtained from the cyclic loading of the test cable for $\pm 100 \mathrm{~mm}$ deflection. The

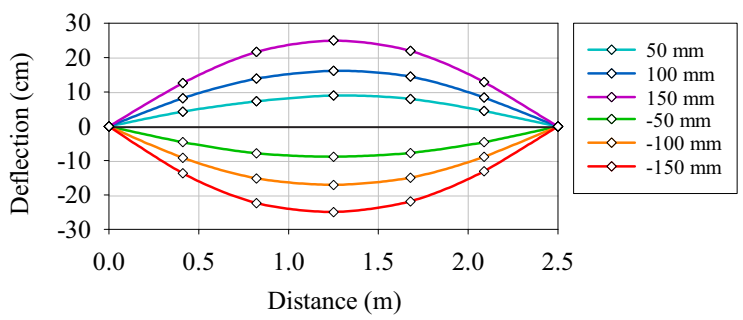

Figure 4. Subsea cable deflection measurement (the diamonds) at five points between the two clamped ends. The solid curves show the second degree polynomial function fitted to the measured data.

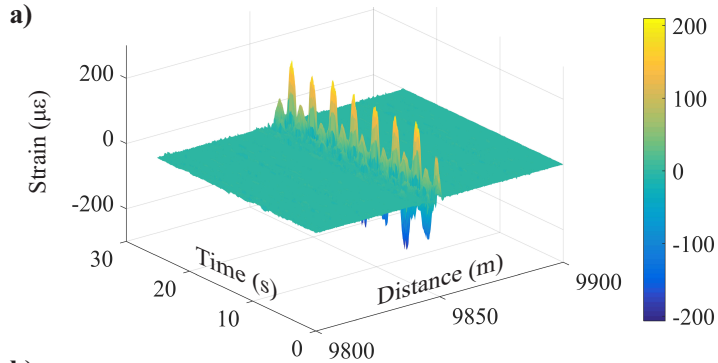

b)

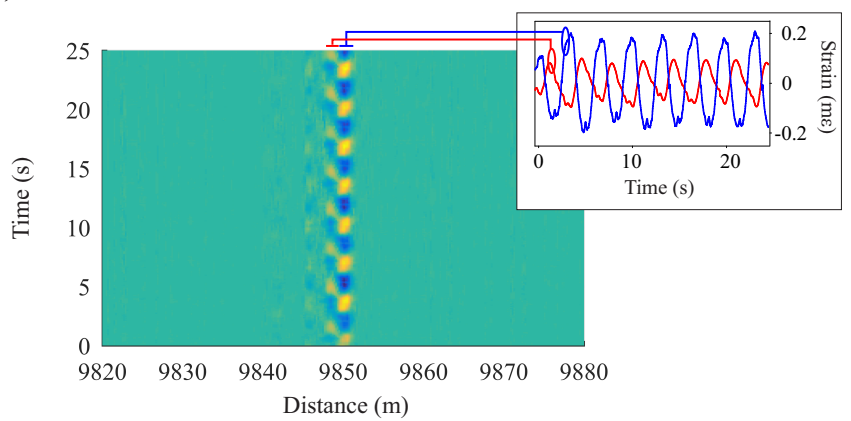

Figure 5. a) The 3D map of the strain distribution along the sensing fibre as a function of time and distance at the far-end of the sensing fibre measured by the DVS system. b) The top view of the 3D result showing two parallel patterns with the same period but with a phase difference of $180^{\circ}$. (Inset: Strain rate at two fixed sampling points on the fibre as a function of time.)

3D diagram of figure 5(a) shows the strain distribution along the sensing fibre as a function of time and distance. A periodic strain variation with an amplitude of $200 \mu \varepsilon$ at the section of the sensing fibre that resides inside the cable can be observed. The diagram also shows that the periodic changes in the strain level is limited to a small portion of the sensing fibre which resides inside the cable.

Figure $5(\mathrm{~b})$ shows the top view of the 3D diagram shown in figure 5 (a). In this diagram, two parallel patterns at the vicinity of $9850 \mathrm{~m}$ can be observed. Both patterns have the same period, but are $180^{\circ}$ out of phase as shown in the inset diagram. It can also be seen that the mid point of the two patterns are $1.6 \mathrm{~m}$ apart.

Figure 6 shows the strain variation at a fixed point on the sensing fibre as a function of time. The three diagrams in this figure show the strain rate for the deflection ranges of $\pm 50 \mathrm{~mm}, \pm 100 \mathrm{~mm}$, and $\pm 150 \mathrm{~mm}$. The circles represent the strain rate measured by the DVS system at a single point on the sensing fibre while the dashed lines represent the strain level experinced by the loose tube that srounds the sensing fibre for an ideal periodic motion of hydraulic actuators.

The dashed lines in this figure were calculated using equation (7). First, the data points on figure 4 were used to measure cable deflection. For $\pm 150 \mathrm{~mm}$ cable displacement, for instance, the following function was obtained by fitting a second degree polynomial to the data points of figure 4

$$
\delta(x)=0.159 x^{2}-0.399 x-0.0036 .
$$

The fitted data had $R^{2}$ value of 0.999 . The curvature of the 
a)

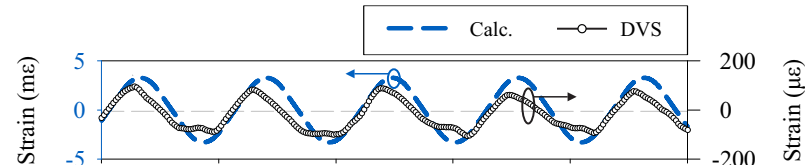

b)

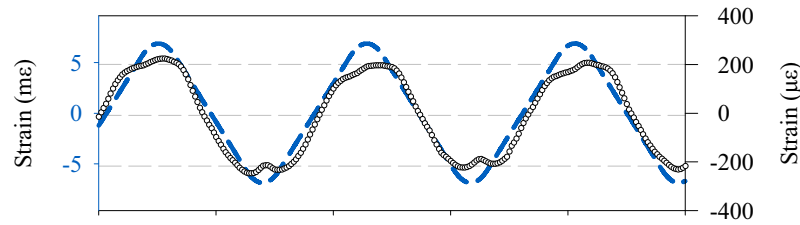

c)

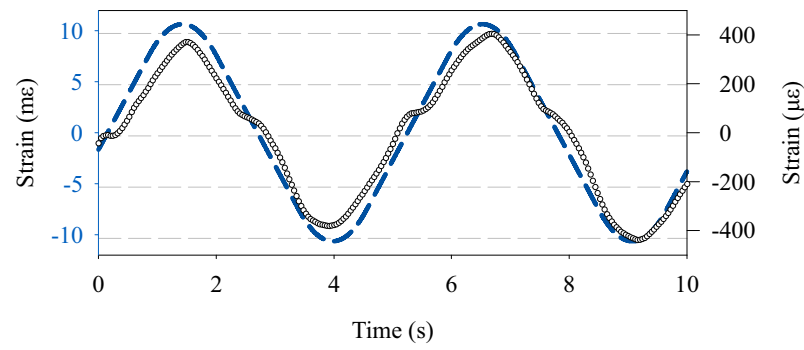

Figure 6. Strain variation at a fixed point on the cable for a) $\pm 50 \mathrm{~mm}$ deflection, b) $\pm 100 \mathrm{~mm}$ deflection, and c) $\pm 150 \mathrm{~mm}$ deflection. The dashed lines show the strain level experienced by the loose tube that surrounds the optical fibre. The strain level on the loose tube was calculated using the deflection measurement of the cable. The circles represent the strain level on the sensing fibre measured by the DVS system.

cable was calculated by replacing equation (8) in (5):

$$
\kappa(x)=\frac{2 \times 0.159}{\left[1+(2 \times 0.159 x-0.399)^{2}\right]^{3 / 2}} .
$$

The optical fibre elongation was then calculated by replacing the curvature function $\kappa(x)$ and other cable parameters in equation (7) and numerically solving the resulting equation. The distance between the principal axis of the cable and the optical fibre was $d=75 \mathrm{~mm}$. Finally, to obtain the bending strain on the cable shown by the dashed lines in figure 6 , the cable elongation was divided by the gauge length of the DVS system which was set to $1 \mathrm{~m}$.

According to figure 6(a), for $\pm 50 \mathrm{~mm}$ deflection, a subcomponent of the cable at distance $d=75 \mathrm{~mm}$ from the cable principal axis experiences a periodic strain with an amplitude of $0 \sim 3.3 m \varepsilon$. The corresponding strain excursion measured by the DVS system was $96 \mu \varepsilon$. For $\pm 100 \mathrm{~mm}$ and $\pm 150 \mathrm{~mm}$ deflections (figure 6(b) and (c)), the subcomponents experiences a strain with an amplitude of $6.88 \mathrm{~m} \varepsilon$ and $10.65 \mathrm{m \varepsilon}$ while the DVS system measured a strain excursion of $232 \mu \varepsilon$ and $415 \mu \varepsilon$, respectively.

Figure 7 shows the test results for the impact test. Figure 7 (a) shows that the weight has impacted the cable at $9851 \mathrm{~m}$ away from the front end of the fibre at $t=2.115 \mathrm{~s}$ after triggering the DVS system. Figure 7(b) exhibit a 2D crosssection of figure 7(a) at the point of impact as a function of time. According to this diagram, the optical fibre experiences a strain variation of $-139.8 \mu \varepsilon$ to $169.1 \mu \varepsilon$ as a result of the impact. The cable oscillation following the impact can also be observed in this plot.
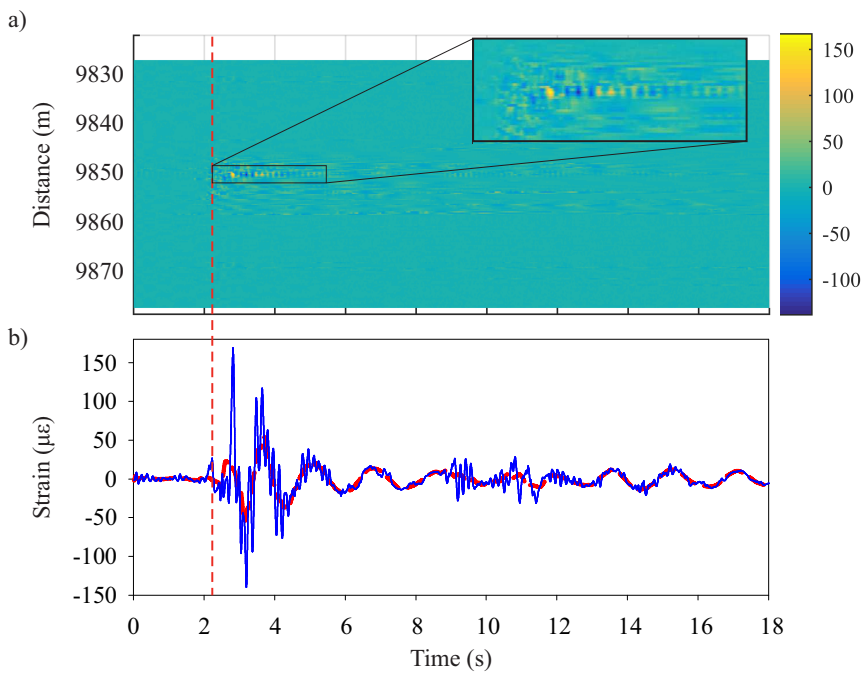

Figure 7. The impact test results. a) The strain distribution map of the sensing fibre as a function of time and distance. The color-bar in this diagram shows the strain level in $\mu \varepsilon$. b) $2 \mathrm{D}$ cross section of the $3 \mathrm{D}$ diagram at the point impact demonstrating the evolution of strain level as s function of time following the impact of a $4 \mathrm{~kg}$ weight. The solid blue line represents the strain level experienced by the fibre embedded in the cable at the point of impact. It consists of a low and a high frequency vibration. The red dashedline represents the filtered data at the same position demonstrating the low frequency vibration of the cable.

\section{Discussion}

The location of the oscillation shown in figure 5(a) matches the position at which the $10 \mathrm{~km}$ dummy sensing fibre was coupled to the fibre inside the subsea cable. Moreover, the phase and frequency of the oscillation agree with that of the hydraulic actuators. This figure shows that the periodic changes in the strain level is limited to a small section of the sensing fibre and that there is no cross-talk between the strained and un-strained regions of the fibre. From the top view of the $3 \mathrm{D}$ diagram, two periodic patterns with the same frequency but with $180^{\circ}$ phase difference can be distinguished. This response can be explained with the help of figure 8 This figure shows the axial section of a deflected cable and its constituent elements at a fixed time. From this figure, it can be seen that the fibre within measurement section 1 undergoes compression while that of measurement section 2 experiences tension. Therefore, while the strain level over section 1 will be at its minimum value, the strain level over section 2 will

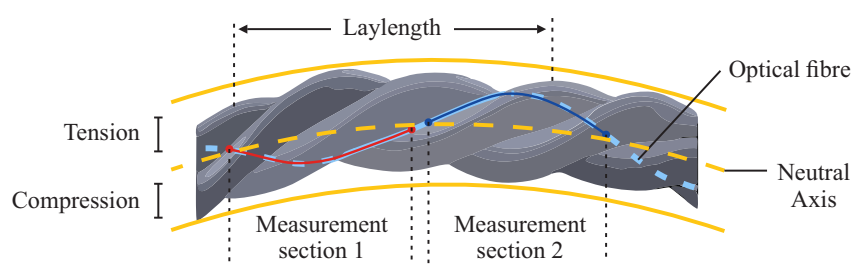

Figure 8. The axial section of a deflected cable and its constituent elements at a fixed time. The sensing fibre in section 1 (distinguished by a red line) experiences compression while the fibre in section 2 (distinguished by a blue line) undergoes tension. 
be at its maximum. Therefore, the strain measurement from two adjacent sections of the fibre will have opposite signs as can be observed in figure 5(b). The distance over which the sign of the strain level flips from positive to negative and vice versa depends on the lay length of the cable as indicated in figure 8

This behaviour agrees with the optical fibre elongation analysis of equation (7). The numerical analysis showed that if a $2.5 \mathrm{~m}$ cable with a lay-length of $1.6 \mathrm{~m}$ is interrogated using a DVS system with $1 \mathrm{~m}$ spatial resolution, two strain patterns with opposite signs will be observed since part of the fibre experiences compression while the adjacent section of the fibre experiences tension. It should be pointed out that $1.6 \mathrm{~m}$ separation between the two patterns matches the lay-length of the test cable.

Figure 6 shows that the frequency and phase of the induced strain at a single point on sensing fibre matches that of the hydraulic actuators for all three deflection ranges. The results in the three diagrams of figure 6 indicate that the strain measured by the DVS system in only a fraction of the axial strain of the cable. For $\pm 50 \mathrm{~mm}$ deflection, for instance, the optical fibre inside the cable experiences $2.9 \%$ of the total axial strain. The level of coupling between the sensing fibre and cable increases to $3.3 \%$ and $3.8 \%$ of the total strain for $\pm 100 \mathrm{~mm}$ and $\pm 150 \mathrm{~mm}$ deflections, respectively. The difference between the measured and actual strain is due to the weak mechanical coupling between the optical fibre and the rest of the cable outer serving. As briefly discussed in section III. subsea power cables contain a loose tube optical fibre bundle used for communication purposes. The loose tube fibre bundles are designed to mechanically decouple the optical fibres and the cables in order to eliminate the environmental effects on the optical signals propagating inside the fibres. This mechanical decoupling explains why the DVS system used in this study only measures a fraction of the total bending strain on the test cable.

These diagrams also show that the pattern of the strain variation along the fibre deviates from the calculations. This deviation has two reasons:

1) The dashed line in the diagram is drawn with an assumption that the hydraulic actuators oscillate in perfect triangular wave. In reality, their movement were not as smooth and had a notable pause before the change of direction. These effects can be observed in the strain pattern measured by the DVS.

2) The second source of discrepancy between the calculated and measured strain is due to the geometry of the cable. The dashed lines in figure 6 show the bending strain at a fixed distance from the principal axis of the cable while the sensing fibre measures the net strain level due to both bending and tensile strain over a certain gauge length.

Figure 7(a) shows a localized strain variation with $1 \mathrm{~m}$ spatial resolution demonstrating the location of the impact. Unlike the cyclical loading diagrams of figure 5 there is no antiphase strain pattern in parallel since the impact-induced strain does not spread far from the location of the impact. Figure 7.b) shows that following the initial impact, the amplitude of the strain attenuates exponentially. The high (solid blue line) and low (dashed red line) frequency oscillation observed in this diagram demonstrates the interaction between the testweight and the cable from the first impact and the subsequent bounces of the weight of the cable. According to this diagram, the minimum strain sensitivity of the DVS system used in this study is $4 \mu \varepsilon$. The higher noise level measured in this study was mainly due to $1 / f$ noise which is the dominant noise source at low frequencies. In addition, the test was carried out at the end of a $10 \mathrm{~km}$ fibre which reduced the SNR further.

\section{CONCLUSION}

In this study, it was shown that DVS systems are capable of fully quantifying dynamic strains along the optical fibre embedded in a subsea cable with a high spatial and strain accuracy. It was shown that the sensing fibre experiences less than $4 \%$ of the bending strain due to the helical geometry of the optical fibre inside the cable as well as the provisions implemented to mechanically decouple the fibre from the cable. Therefore, the optical fibre can be used to estimate the dynamic strains experienced by the subsea cable during the installation phase for any sign of excessive tension as well as post installation to monitor potential damages due to human activities in marine environment such as fishing and ship anchoring. The sensing unit demonstrated in this study had a $10 \mathrm{~km}$ sensing range. This technology can be improved to achieve more than an order of magnitude improvement in the sensing range and sensitivity by incorporating an in-line optical amplifier and using a bespoke cable design with a higher mechanical coupling between the optical fibre and the outer serving.

\section{REFERENCES}

[1] B. Snyder and M. Kaiser, "Ecological and economic cost-benefit analysis of offshore wind energy", Renew Energy., vol. 34, no. 6, pp. 1567-1578, 2009.

[2] M. Esteban, J. Diez, J. López and V. Negro, "Why offshore wind energy?", Renew Energy., vol. 36, no. 2, pp. 444-450, 2011.

[3] "Export Cable Reliability - Description of Concerns", Offshore Renewable Energy Catapult, 2017. [Online] Available: https://goo.gl/XDj3mK.

[4] "Competition to improve subsea cabling systems - Carbon Trust", Carbon Trust, 2018. [Online]. Available: https://goo.gl/Czy6fj .

[5] T. Worzyk, "Damages and Repair," in Submarine Power Cables. Berlin: Springer Berlin, 2013, pp. 211-236.

[6] H.S. Yoon and W.B. Na, "Safety assessment of submarine power cable protectors by anchor dragging field tests," Ocean Eng., vol. 65, pp. 1-9, 2013.

[7] A. Masoudi and T. P. Newson, "Contributed Review: Distributed optical fibre dynamic strain sensing", Rev. Sci. Instrum., vol. 87, no. 1, p. 011501, 2016.

[8] Y. Muanenda, "Recent Advances in Distributed Acoustic Sensing Based on Phase-Sensitive Optical Time Domain Reflectometry", Journal of Sensors, vol. 2018, p. 3897873, 2018.

[9] K. Song, M. Kishi, Z. He and K. Hotate, "High-repetition-rate distributed Brillouin sensor based on optical correlation-domain analysis with differential frequency modulation", Opt. Lett., vol. 36, no. 11, p. 2062-2064, 2011.

[10] A. Masoudi, M. Belal, and T. P. Newson, "Distributed dynamic large strain optical fiber sensor based on the detection of spontaneous Brillouin scattering," Opt. Lett., vol. 38, no. 17, pp. 3312-3315, 2013. 
[11] B. Li, L. Luo, Y. Yu, K. Soga, and J. Yan, "Dynamic strain measurement using small gain stimulated Brillouin scattering in STFT-BOTDR," IEEE Sensors J., vol. 17, no. 9, pp. 2718-2724, 2017.

[12] M. J. Shangguan, C. Wang, H. Xia, G. Shentu, X. Dou, Q. Zhang, and J. W. Pan, "Brillouin optical time domain reflectometry for fast detection of dynamic strain incorporating double-edge technique," Opt. Commun., vol. 398, pp. 95-100, 2017.

[13] Y. Mizuno, N. Hayashi, H. Fukuda and K. Nakamura, "Phase-detected Brillouin optical correlation-domain reflectometry", Opt. Rev., vol. 25, no. 3 , pp. $473-485,2018$

[14] A. Masoudi and T. P. Newson, "High spatial resolution distributed optical fiber dynamic strain sensor with enhanced frequency and strain resolution," Opt. Lett., vol. 42, no. 2, pp. 290-293, 2017.

[15] X. Fan, G. Yang, S. Wang, Q. Liu, and Z. He, "Distributed fiber-optic vibration sensing based on phase extraction from optical reflectometry," J. Lightwave Technol., vol. 35, pp. 3281-3288, 2017.

[16] S. Liehr, Y. Muanenda, S. Münzenberger and K. Krebber, "Relative change measurement of physical quantities using dual-wavelength coherent OTDR", Opt. Express, vol. 25, no. 2, pp. 720-729, 2017.

[17] S. Liehr, S. Münzenberger, and K. Krebber, "Wavelength-scanning coherent OTDR for dynamic high strain resolution sensing," Opt. Express, vol. 26, no. 8, pp. 10573-10588, 2018.

[18] G. Yang, X. Fan, Q. Liu and Z. He, "Frequency Response Enhancement of Direct-Detection Phase-Sensitive OTDR by Using Frequency Division Multiplexing", J. Lightwave Technol., vol. 36, no. 4, pp. 1197-1203, 2018.

[19] A. H. Hartog, "Rayleigh Backscatter: Distributed Vibration Sensors and Static Measurements," in An Introduction to Distributed Optical Fibre Sensors, Boca Raton, FL: CRC Press, 2017, pp. 379-398.

[20] A. Masoudi and T. P. Newson, "Analysis of distributed optical fibre acoustic sensors through numerical modelling", Opt. Express, vol. 25 , no. 25, p. 32021-32040, 2017.

[21] J. Q. Bi and P. Anliker "Twisted pair loose tubes: A new design of fiber optic cables", in 12th Annual Conference on European Fibre Optic Communications and Networks, Heidelberg, Germany, Jun. 1994, pp. 23-26.

[22] D. J. Walton and D. S.Meek, "Curvature extrema of planar parametric polynomial cubic curves," J Comput Appl Math., vol. 134, pp. 69-83. 2001.

[23] J. M. Gere, "Stresses in Beams (Basic Topics)," in Mechanics of Materials, 6th ed. Belmont, CA: Thomson Learning, 2004.

[24] A. Masoudi, M. Belal, and T. P. Newson, "A distributed optical fibre dynamic strain sensor based on phase-OTDR." Meas. Sci. Technol., vol. 24, no. 8, p. 085204, 2013. 